\title{
EVALUACIÓN ESTRATÉGICA DE LAS ORGANIZACIONES ORIENTADAS A PROYECTOS
}

\author{
Strategic Evaluation of Project Management Organizations
}

\section{EPISTEMUS}

ISSN: 2007-8196 (electrónico)

ISSN: 2007-4530 (impresa)

Anié Bermudez Peña ${ }^{1}$

Roberto Delgado Victore 2

Gilberto Fernando Castro Aguilar 23

Rosa María Renté Labrada 4

Noichel Juan Hernández 5

Recibido: 14 de octubre de 2018,

Aceptado: 13 de noviembre de 2018

Autor de Correspondencia:

Dra. Anié Bermudez Peña

Correo:abp@uci.cu

\section{Resumen}

El proyecto constituye la unidad básica organizativa de las empresas que inciden de manera decisiva en su progreso. La Dirección Integrada de Proyectos permite el desarrollo de buenas prácticas, mejora continua, gestión del cambio, integración y sinergia empresarial. La política de dirección por proyectos, el uso de indicadores y las tendencias en un proceso estratégico integrado de dirección son la base fundamental para el desarrollo de la Inteligencia Empresarial. El trabajo tiene como objetivo el desarrollo de un modelo de evaluación estratégica para las empresas que trabajan por proyectos en la DIP orientadas al Modelado de Información de Construcción. A través de la aplicación de un modelo de evaluación estratégica en el ciclo de vida de la inversión, se permite compartir el comportamiento de los indicadores en los cortes estratégicos entre directivos, equipo y partes interesadas, para proceder a la toma de decisiones en un proceso integrado de dirección. La aplicación de técnicas de computación blanda dota a los sistemas informáticos de robustez, eficiencia y adaptabilidad para apoyar el control de la ejecución de proyectos, manejar adecuadamente la incertidumbre en los datos, así como realizar un diagnóstico y pronóstico de los proyectos. La Inteligencia Empresarial en las organizaciones que trabajan por proyectos, se desarrolla sobre la base de la información estructurada por indicadores que genera el proyecto en los cortes, para compartirla en la toma de decisiones siguiendo los procesos de mejora y buenas prácticas para garantizar el perfeccionamiento empresarial.

Palabras clave: Computación blanda, Dirección Integrada de Proyectos, Inteligencia Empresarial, Modelado de Información de Construcción.

\section{Abstract}

Project constitutes the basic organizational unit of the companies that have a decisive influence on its progress. Integrated Project Management allows the development of good practices, continuous improvement, change management, integration and business synergy. Project management policy, the use of indicators and trends in a strategic integrated management process are the fundamental basis for the development of business intelligence. The objective of this work is the development of the strategic evaluation of the organizations that work for projects in the DIP oriented to Building Information Modeling. Through the application of a strategic model in the investment life cycle, it is allowed to share the indicators behavior in the strategic cuts between managers, team and stakeholders, to proceed to the decision making in integrated process management. Application of soft computing techniques equips computer systems with robustness, efficiency and adaptability to support the project execution control, adequately handle data uncertainty, as well as perform a projects diagnosis and forecast. Business intelligence in project management organizations is developed on basis of the information structured by indicators generated by the project, to share it in decision making following the improvement processes and good practices to ensure business improvement.

Keywords: Building Information Modeling, Business Intelligence, Project Management Organizations, Soft computing.

1 Departamento de Gestión de Proyectos, Universidad de las Ciencias Informáticas. \{abp, robertodv\}@uci.cu

2 Facultad de Ingeniería, Universidad Católica Santiago de Guayaquil, Ecuador. gilberto.castro@cu.ucsg.edu.ec

3 Facultad de Ciencias Matemáticas y Físicas, Universidad de Guayaquil, Ecuador. gilberto.castroa@ug.edu.ec

4 Facultad 2, Universidad de las Ciencias Informáticas, La Habana, Cuba. rrente@uci.cu

5 Facultad 1, Universidad de las Ciencias Informáticas, La Habana, Cuba. njuan@uci.cu 


\section{INTRODUCCIÓN}

La obtención de los resultados de las investigaciones orientadas a la evaluación de las organizaciones que trabajan la dirección por proyectos, ha transitado por períodos prolongados entre su obtención y su aplicación. El desarrollo actual de la sociedad con su creciente necesidad de soluciones a los problemas de su desarrollo, requiere de la generalización de los conocimientos de la gestión empresarial, en el menor plazo de tiempo posible, apoyada por la Inteligencia Empresarial a través de un modelo de evaluación estratégica integrado.

Constituye un reto actual, acortar este período de tiempo de aplicación con el objetivo de compartirlo y convertirlo en un producto socialmente útil. Las Tecnologías de la Informática y las Comunicaciones (TICs), las base de datos y el ciclo de gestión de conocimiento asociado a la dirección por proyecto, permiten desarrollar el conocimiento implícito en los proyectos, almacenar la información, gestionar el conocimiento y compartirlo con quienes necesitan hacer uso del mismo, para lograr el mejoramiento continuo de la calidad del proceso de dirección en las empresas en perfeccionamiento (Delgado, 2011).

El conocimiento transita por un proceso objetivo, se desarrolla independientemente que se dirija o no. Con la aplicación de un modelo estratégico de gestión del conocimiento, es posible evaluar su comportamiento e incidencia en el desarrollo de los proyectos, identificar sus regularidades, desarrollar un diagnóstico y una proyección con el objetivo de dirigir su proceso de desarrollo. Con dicho modelo se estará en condiciones de compartir el conocimiento y ponerlo en función del beneficio del equipo de proyecto y las partes interesadas, a través de una eficiente capacitación del hombre que dirige el mismo, con el apoyo de las TICs en el marco de la Inteligencia Empresarial. Este trabajo tiene como objetivo desarrollar un modelo de evaluación estratégica para la gestión de proyectos, que permita evaluar el desempeño de las empresas y compartir el mismo con los directivos y las partes interesadas, en un proceso de mejora continua aplicando los conceptos de la Inteligencia Empresarial.

En el contenido se muestran los antecedentes, el modelo para el ciclo de gestión de proyectos, su impacto, el desarrollo de la Inteligencia Empresarial en la DIP (Delgado, 2011) y su orientación al BIM (Holness, 2008). Se aplican técnicas de soft computing y aprendizaje automático para la implementación del modelo en un entorno real de aplicación. Al final se muestran las conclusiones y recomendaciones.

\section{Materiales y métodos}

El desarrollo empresarial es un proceso objetivo, que se realiza en las organizaciones de forma espontánea o a través de un proceso de gestión del conocimiento dirigido. Cuando el cambio se desarrolla a través de un proyecto de cambio dirigido, se hace uso de tecnologías con una estrategia concebida en un proceso de dirección, donde la efectividad y los resultados se alcanzan en menos tiempo, en el marco del presupuesto y con la calidad requerida por el cliente y las partes interesadas.

En los conceptos fundamentales tenemos el modelo

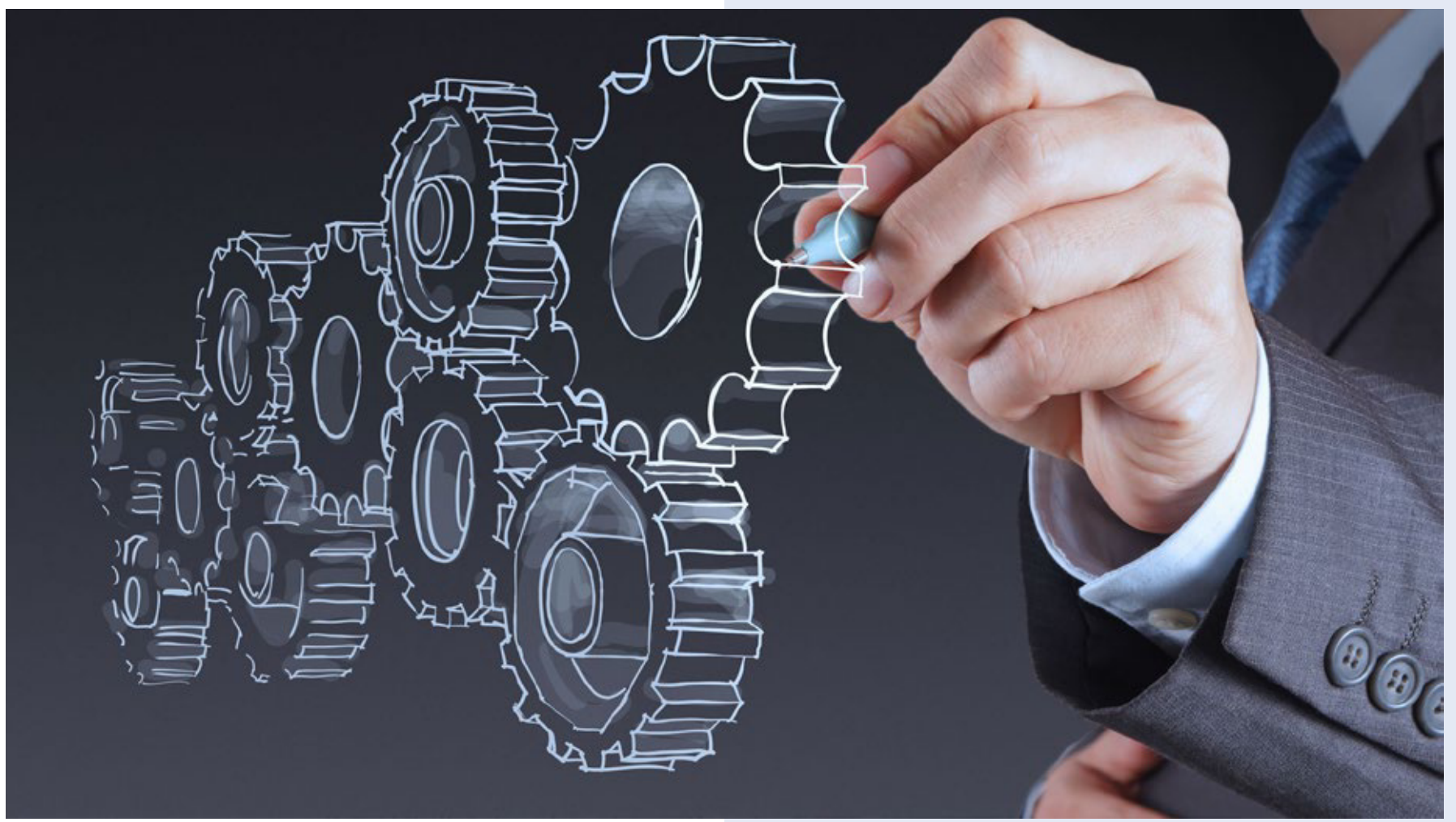


empresarial, la gestión económica financiera como forma de evaluar un programa de proyectos en distintas fases de desarrollo, la gestión del conocimiento de la organización y el recurso humano, como medio de garantizar la mejora continua y el perfeccionamiento, con su sistema de información y comunicación en un proceso integrado en el ciclo de vida de los proyectos de inversiones (Delgado, 2009).

El Modelado de Información de Construcción (BIM), es el sistema de información integrado, estratégico, único y multidimensional para la gestión de los proyectos de la construcción (Holness, 2008). Se basa en un modelo virtual en 3D donde se prepara el proyecto antes de su ejecución. Permite la integración continua como forma de elevar la calidad de la preparación, para garantizar ejecuciones efectivas y sostenibles, desde la pre-inversión hasta su demolición. Es la metodología que permite la representación de documentos gráficos y escritos, con características físicas y funcionales, desarrollada para proyectos integrales, por organizaciones maduras, con un importante apoyo informático y un protocolo que regula su funcionamiento.

El perfeccionamiento empresarial requiere del uso de tecnologías de avanzada en términos de dirección, con el objetivo de garantizar un desarrollo armónico entre el uso del equipamiento de alto desarrollo, las nuevas tecnologías, los sistemas informáticos actualizados y una alta calificación del potencial humano. No es posible dirigir equipos modernos de proyectos BIM, con sistemas de dirección obsoletos. La Dirección Integrada de Proyectos (DIP) apoyada por los sistemas informáticos profesionales como GESPRO, MS Project, los complementos del Office y Primavera, haciendo uso de la Inteligencia Empresarial, ha alcanzado un alto desarrollo, tanto en el ámbito nacional como internacional. La capacitación del personal, que de una forma $u$ otra labora asociado a estas tecnologías requiere de una atención personalizada de capacitación continua, en función de las necesidades del conocimiento de cada nivel de Inteligencia Empresarial, con el objetivo de lograr un alto desempeño en el desarrollo de sus funciones.

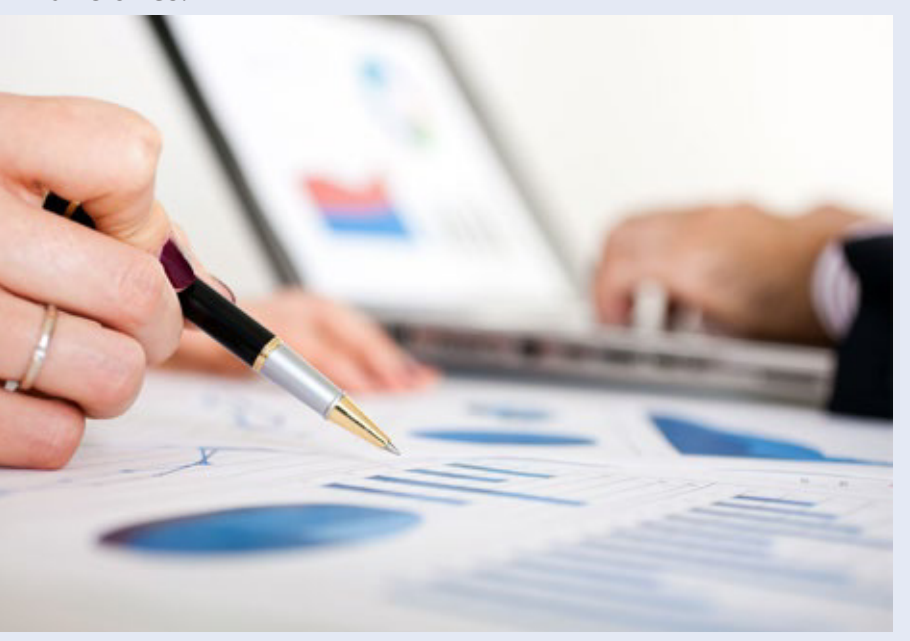

\section{Modelo DIP orientado al BIM}

El modelo tiene su inicio en el protocolo que permite regular todas sus funciones a lo largo del ciclo de vida de la inversión. A partir de una estructura funcional garantiza el desarrollo de los procesos clave, donde se generan los valores a través de la aplicación de los activos de las áreas de conocimiento. Los sistemas de gestión y apoyo, los procesos estratégicos en función de la misión y la visión de la organización, en un sistema integrado de dirección, garantizan los intereses del cliente, en un proceso de mejora continua y perfeccionamiento del sistema (SEI, 2010). Se utiliza una base de datos de las inversiones en proceso y terminadas como base para la gestión del conocimiento.

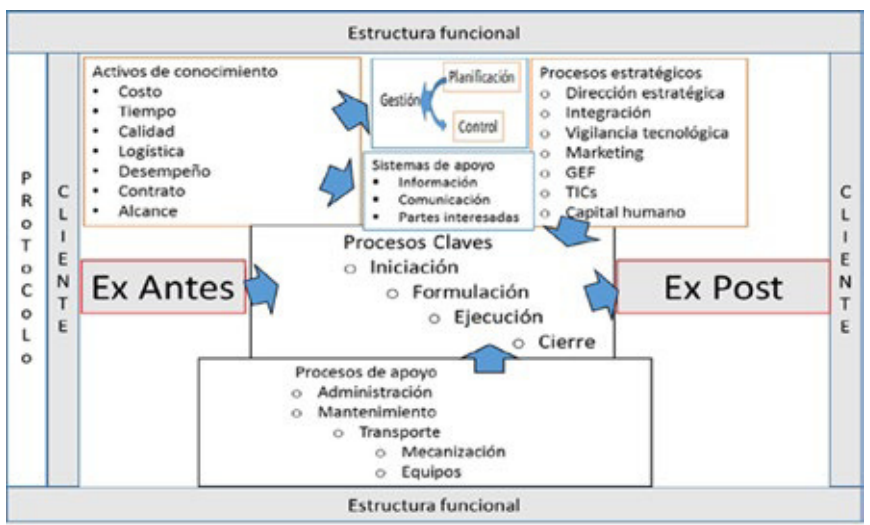

Figura 1. El Modelo DIP orientado al BIM.

La estructura funcional se desarrolla sobre la base de una organización matricial donde los proyectos representan la unidad básica del proceso de dirección. La estructura se apoya en el modelo que tiene como inicio el protocolo que rige todo el proceso conceptual de dirección. El desarrollo de los procesos clave genera el conocimiento tomando como base los contenidos de los activos de conocimiento de la organización o áreas de conocimientos alimentadas por los procesos estratégicos y los procesos de apoyo.

El director de la empresa tiene como función principal la supervisión de los directores de proyectos a través del director de producción, por ser los actores principales del sistema de dirección.

Las áreas de la estructura funcional tienen un carácter metodológico sobre los directores de proyectos asesorando todo el desarrollo de los proyectos con la aplicación actualizada de los contenidos definidos en los activos de conocimiento de la organización. Es la encargada de evaluar los nuevos conocimientos que se generan en los procesos clave, a través de la gestión documental, para actualizar los contenidos de los activos de conocimiento de la organización, recogiendo las buenas prácticas y las lecciones aprendidas (Amendola, 2004). El modelo de gestión del conocimiento de la DIP, garantiza en los procesos de cierre la actualización de la base de 


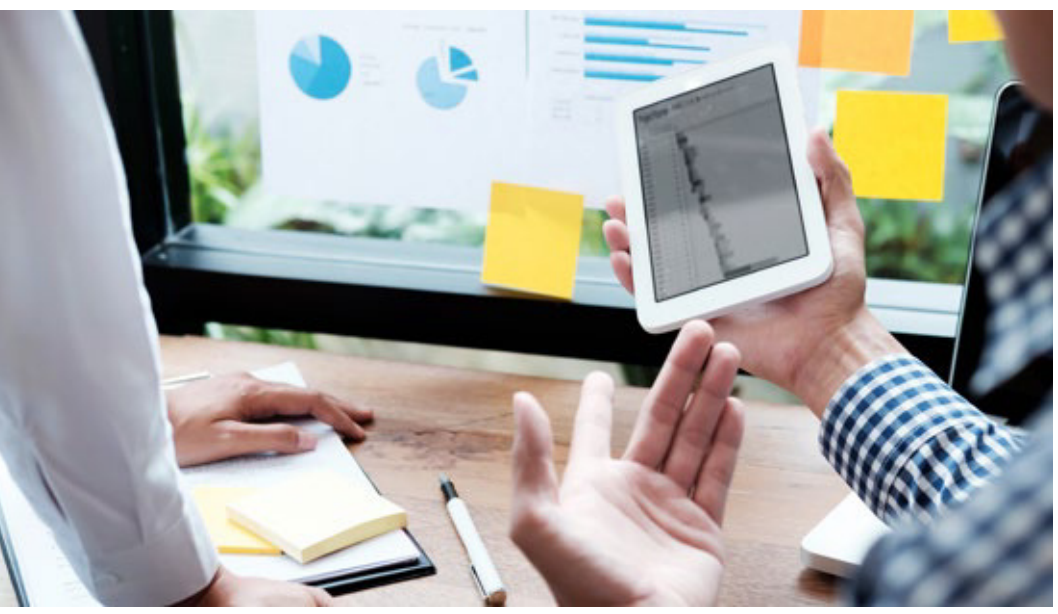

datos de proyectos terminados como medio de garantizar la retroalimentación del sistema y su perfeccionamiento.

El director de la empresa, que dirige por proyecto, se reúne con los interesados en un proyecto priorizado para evaluar su desempeño después de haber entrado al sistema de información para actualizar la situación del mismo y precisar los problemas más importantes, para con la estructura funcional evaluar la proyección estratégica del mismo. Por otra parte, controla la mejora continua y el perfeccionamiento que desarrolla la estructura funcional en la captación de las buenas prácticas de los procesos clave y el perfeccionamiento de los activos de conocimientos de la organización.

\section{Sistema estratégico integrado de dirección}

Tomando como base las necesidades del entorno y el desarrollo alcanzado en la aplicación de la DIP, se hace necesario el uso de una herramienta que permita la instrumentación de la misma. En el contenido se desarrolla un procedimiento donde se integra la dirección estratégica, la dirección por objetivos, la planificación con enfoque sistémico, la dirección de la calidad, el uso de sistemas de costos avanzados, la contabilidad, la aplicación de las TICs, el uso de los valores y la logística (Torres, 2008), tomando como base un sistema informático capaz de garantizar la gerencia por proyectos y potenciar su uso en las empresas. El sistema informático permite la aplicación de los algoritmos de optimización, evaluación de variantes, análisis de costo y tiempo. Se obtiene el diagrama de barras, la curva de distribución de recursos, los balances, el fondo de recursos compartidos y el estudio logístico para garantizar el suministro en función de la programación, para alcanzar los resultados previstos en el marco del presupuesto, acortando los plazos de ejecución con la calidad requerida.

En el proceso del control de ejecución, se desarrolla la evaluación del proyecto, con técnicas de comportamiento, diagnóstico y pronóstico, que brindan los elementos para la toma de decisiones y una gestión de proyecto efectiva a nivel de empresa, integrando la estructura funcional en apoyo al logro de los resultados de los proyectos.

\section{Método del eslabón más débil}

El proceso de dirección por proyectos se presenta por cuatro subprocesos principales iniciación, planificación, ejecución y cierre, estos pueden ser estructurados de acuerdo con sus respectivos contenidos. El método del eslabón más débil se basa en una organización previa del proceso de análisis, un mapeo del proceso donde se definen sus módulos, su estructura y dependencias. En cada módulo se definen los indicadores a evaluar, su comportamiento a través de las tendencias de los indicadores y el estudio ponderado de los mismos para establecer las prioridades de perfeccionamiento de los mismos. Selección del eslabón más débil del proceso con sus indicadores, el estudio de causa efecto y la estrategia de perfeccionamiento.

El proceso identifica el eslabón más débil y permite establecer las prioridades según el comportamiento de los indicadores para una sucesión de acciones. El proyecto de cambio como forma de dirección del perfeccionamiento es un proceso de la Inteligencia Empresarial apoyado en el conocimiento que aporta PMBOK (PMI, 2017), Prince (Prince, 2009), MS Project y las tecnologías del software libre. El cierre del ciclo permite su perfeccionamiento en función de las prioridades dadas por los indicadores. La Figura 2 muestra la secuencia propuesta para el desarrollo del método asociado a la cadena de valores, la ingeniería del valor, la contabilidad y las estrategias de perfeccionamiento. El método del eslabón más débil aplicado sistemáticamente sobre una base del uso de los indicadores permite identificar los problemas más importantes y proceder a sus soluciones.

\section{Método el eslabón más débil}

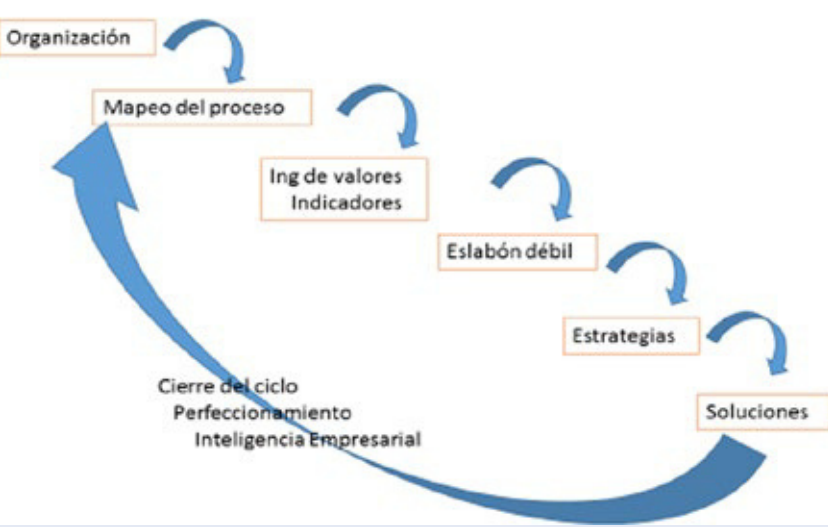

Figura 2. Método del eslabón más débil.

\section{Gestión económica financiera de la organización}

La gestión económica financiera de un proyecto es la base para el desarrollo de un programa de proyectos, en el que los proyectos tienen calendarios diferentes y los procesos clave desarrollan su gestión económica financiera según la fecha de corte del programa. La Figura 3 muestra una representación de un programa con varios proyectos 


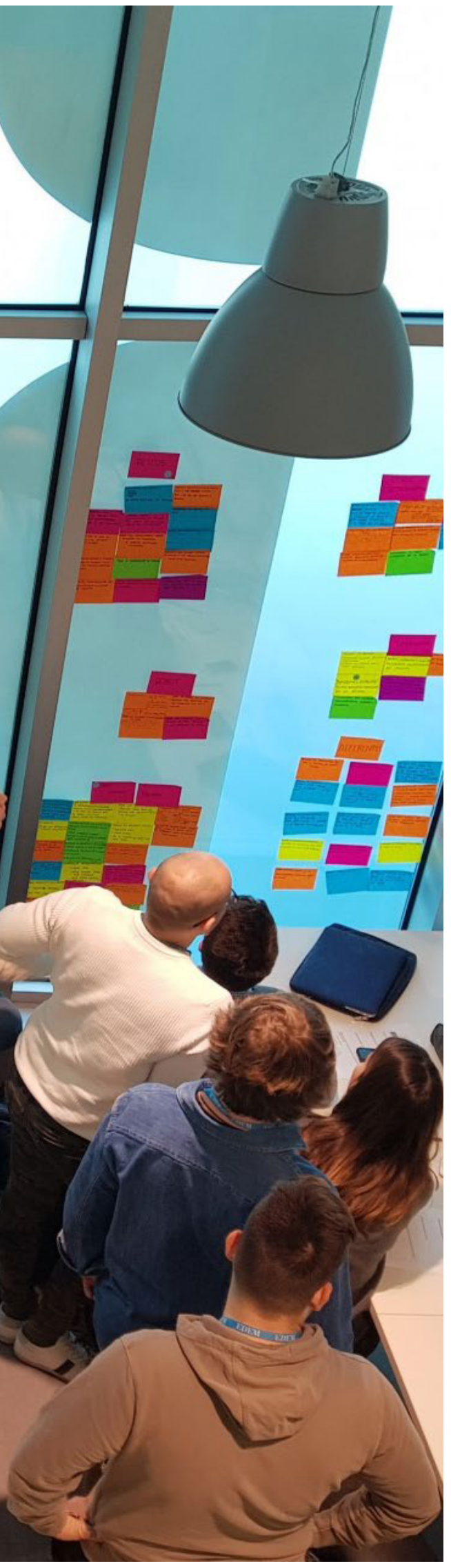

(Pi) y un corte donde cada proceso clave desarrolla su gestión económica financiera según el proceso en que se encuentre, donde por ejemplo el P1 está en ejecución y el P2 en formulación. Se muestra también, la forma de representar un programa con un conjunto de sub-proyectos.
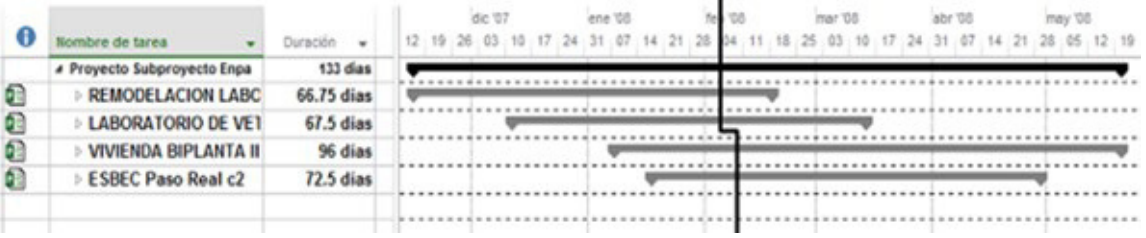

Figura 3. Corte de un programa con proyectos en diferentes procesos claves.

En el corte es necesario precisar cuál es el aporte en cada uno de los proyectos según el proceso clave actual, atendiendo a los resultados de la aplicación del método del valor ganado con la variación del costo en el corte.

La entrada en valores de la organización que dirige un programa de proyectos, con diferentes estados de ejecución, que requieren de un balance en una fecha de corte, está dada por la suma de las variaciones acumuladas del precio de venta de los entregables en cada proyecto. Este valor viene afectado en cada caso por otras acciones asociadas a la gestión del cambio, no conformidades, transacciones comerciales, gestiones bancarias, penalizaciones y demás gestiones económicas, como imprevistos recogidos en los cortes.

Es posible conocer para cada proyecto el costo esperado final y la variación actual del costo. Como los calendarios son diferentes, estas informaciones pueden ser útiles, pero es más importante la evaluación en el corte con el valor ganado, dado que determina la liquides de la organización y la toma de decisiones del sistema de dirección.

\section{Modelo de gestión del conocimiento}

La empresa de alto desempeño se apoya en un conjunto de conceptos tendientes a la aplicación de la Inteligencia Empresarial, que permiten identificar su tránsito desde el estado actual a través de un estudio de comportamiento y diagnóstico, hasta alcanzar una etapa de desarrollo superior de perfeccionamiento. La caracterización de una empresa a través de un conjunto de indicadores ponderados con sus líneas de tendencia, permite conocer el estado de la misma, en su tránsito continuo al desarrollo en proceso ininterrumpido de la Inteligencia Empresarial.

El conocimiento transita por una búsqueda de información y un proceso de aprendizaje, en el que las Tecnologías de la Información y las Comunicaciones con las bases de datos de proyectos en ejecución y terminados juegan un papel importante. El modelo de gestión del conocimiento tiene dos momentos claves que garantizan el proceso continuo de perfeccionamiento. El primero es la toma de decisión de aprobación del proyecto técnico, con el diseño conceptual directivo, en el que la comisión evaluadora realiza un análisis de la calidad de la documentación, certifica que el nuevo proyecto recoge las experiencias de los proyectos ejecutados en la base de datos, como una forma de garantizar el proceso continuo de la Inteligencia Empresarial.

El segundo aspecto importante es la evaluación del desempeño de los directores de proyectos que se genera en los cortes de los proyectos reflejados en el informe de estado, disponible en la base de datos de proyectos en ejecución. Este análisis brinda los elementos necesarios para el desarrollo de la capacitación personalizada de los participantes en la DIP. Estos dos aspectos son fuente importante para la mejora continua del proceso de gestión del conocimiento, en el marco de la Inteligencia Empresarial, estimulando la capacitación y el proceso de aprendizaje de los participantes en la ejecución de los proyectos. 
La figura 4 muestra el modelo propuesto. La información y el conocimiento generan más conocimiento cuando se comparte y se generaliza brindando soluciones de mayor calidad.

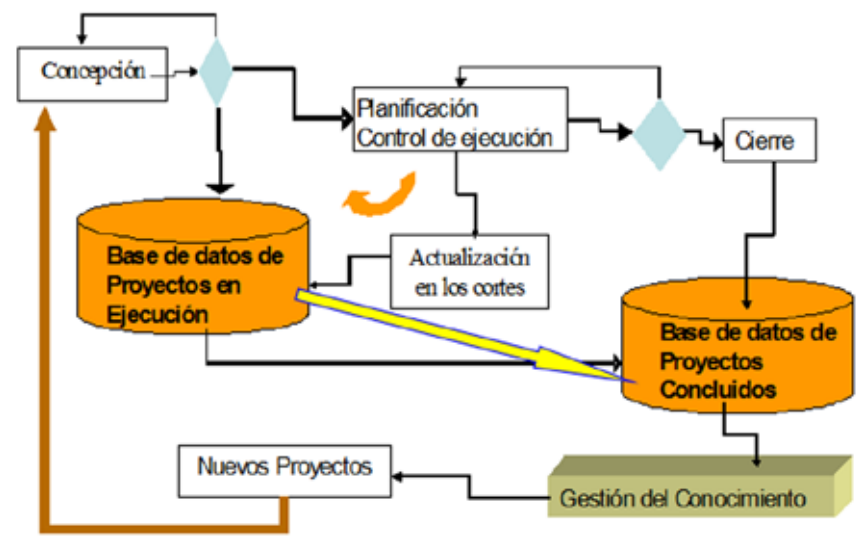

Figura 4. Modelo estratégico para la gestión del conocimiento.

El sistema de información en el BIM representa el medio de garantizar la disciplina, la organización y el control de la documentación a lo largo del ciclo de vida de la inversión. Permite la integración continua y la constructibilidad (Espinosa, 2012), como forma de elevar la calidad de la preparación, para garantizar ejecuciones efectivas y sostenibles. El sistema de información garantiza la actualización continua del As Built con el propósito de facilitar la documentación actualizada de la inversión, en el momento de realizar los mantenimientos especializados programados en el período de explotación, así como todos los ajustes, instrumentación y perfeccionamiento propio del desarrollo del sistema con la visibilidad que brinda en su contexto, por su representación en 3D.

\section{Aplicación de aprendizaje automático y soft computing}

Los avances tecnológicos en la esfera de la gestión de proyectos han posibilitado conocer en un instante la acción que se debe tomar para solucionar determinado problema. Esto se debe gracias a las herramientas informáticas de apoyo a la toma de decisiones, que están fundamentadas en indicadores claros y medibles. El aprendizaje automático se muestra como un campo interdisciplinario donde intervienen: la estadística, la lógica, las matemáticas, las estructuras neuronales, la información teórica, la inteligencia artificial y el soft computing. En las últimas décadas han sido desarrolladas numerosas herramientas para la gestión de proyectos (Stang, 2013).

Varias investigaciones han sugerido soluciones teóricas basadas en aprendizaje automático, minería de datos, inteligencia artificial y soft computing, útiles para la gestión de proyectos. Aplicaciones específicas de estas técnicas en la gestión de proyectos son relativamente pocas en comparación con otras áreas de aplicación. A continuación se citan varios trabajos relacionados.

En (Bath, 2010) se propone un método basado en soft computing para clasificar proyectos en tres clases: simple, medio o complejo. En (Gao, 2010) se construye un sistema de índices para evaluar el desempeño en la gestión de proyectos en cuanto a calidad, costo, tiempo y riesgos. En (Certa, 2010) se propone un sistema de inferencia borroso para el cálculo del indicador que evalúa el rendimiento del proyecto, conjugando dos entradas obtenidas según el análisis del valor ganado: rendimiento de los costos y rendimiento de la planificación. En (Mewada, 2013) se expone un modelo de evaluación de esfuerzo de software aplicando redes neuronales sobre datos con incertidumbre. En (Liu, 2013) se presenta un sistema basado en redes neuronales para la aplicación del modelo de madurez de gestión de proyectos organizacional. En (Govindarajan, 2014) se propone un sistema de inferencia borroso para evaluar la eficiencia en la gestión de proyectos de software. De manera general las investigaciones analizadas no integran las siguientes características:

- Aplicación de técnicas de aprendizaje automático.

- Ajuste del sistema de evaluación según la mejora continua de la organización.

- Integración del modelo con herramientas de gestión de proyectos.

- Implementación sobre software libre.

Aplicación de la solución propuesta sobre la evaluación de la ejecución de proyectos.

\section{Control de la ejecución de proyectos}

A continuación se propone un modelo para el control de la ejecución de proyectos. Este dispone de tres componentes fundamentales: obtener de la base de datos de proyectos terminados los casos necesarios para el aprendizaje, aplicar diferentes técnicas de soft computing y emplear métricas estadísticas de rigor para seleccionar el sistema que mejor evalúe la ejecución de los proyectos en la organización, ver Figura 5. Este mecanismo se realiza

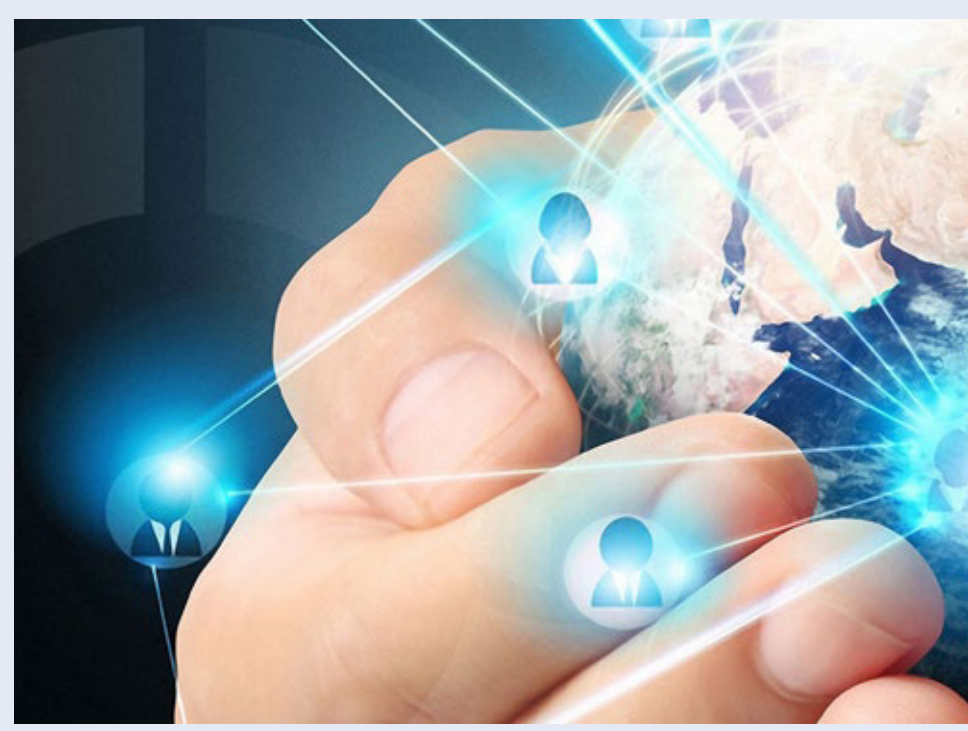


cuando la alta gerencia determina la necesidad de actualizar dicho sistema de evaluación según los cambios producidos por la mejora continua en los estilos de gestión de la organización.

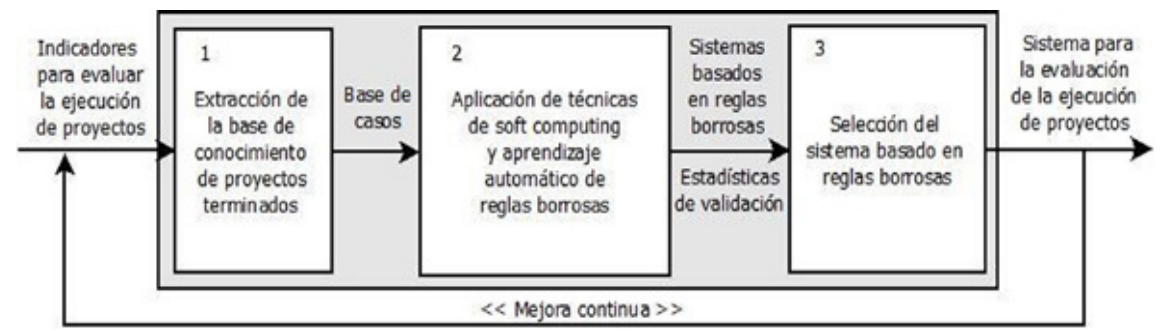

Figura 5. Componentes del método implementado para el control de la ejecución de proyectos.

Para garantizar la mejora continua del sistema propuesto y que este incida positivamente en la adaptación de la organización a los nuevos estilos de gestión, se requiere que se actualice la base de conocimiento de proyectos terminados. Dicha actualización se puede realizar cuando los directivos consideren necesario según la madurez actual de la organización.

En el modelo propuesto, se aplica una estrategia de aprendizaje supervisado que permite ajustar los parámetros graduables de los sistemas de inferencia borrosos, de manera tal que la salida calculada sea lo más parecida a la salida esperada. Para ello es necesario contar con datos de entrenamiento compuestos por patrones de entrada y salida esperada. El aprendizaje se realiza a partir de un conjunto de proyectos terminados, de los cuales se conocen los valores numéricos de los indicadores claves de gestión y la evaluación dada por expertos de la organización. De dicha base de proyectos se extrae el conjunto de casos de entrenamiento y el conjunto de casos de validación.

Se aplican varias técnicas de aprendizaje automático, soft computing e inteligencia artificial, desde la implementación en una biblioteca. Los algoritmos aplicados se basan en técnicas de partición de espacio, redes neuronales, gradiente descendente y algoritmos genéticos. Las técnicas de soft computing aportan a las herramientas informáticas una aproximación al razonamiento humano, a través de la utilización del conocimiento y la experiencia acumulada. Además, permiten ganar en eficiencia, adaptabilidad y equilibrio adecuado entre poder de predicción e interpretación para dar apoyo a la toma de decisiones. Dichas técnicas son robustas ante entornos con entradas ruidosas y tienen una alta tolerancia a la imprecisión de los datos con los que operan; permiten desarrollar soluciones de bajo costo y mayor capacidad de modelación.

\section{RESULTADOS Y DISCUSIÓN}

Se aplican las bases de la Inteligencia Empresarial en un proceso de perfeccionamiento que depende del índice de madurez de la organización, de la capacidad del recurso humano, los medios de trabajo, las fuentes de financiamiento, el medio ambiente y el mercado entre otros aspectos propios de interés.

Mediante un tablero de comando se permite identificar el estado de los indicadores para determinar dónde están las desviaciones para la búsqueda de las causas y brindar las soluciones estratégicas a las mismas. La figura No 5 muestra un corte del proyecto con los indicadores evaluados según su avance. El cuadro de comando de la empresa se alimenta del tablero de comando con la evaluación de los indicadores de los proyectos para realizar la evaluación de la organización. Los indicadores permiten la evaluación de las tareas, subtareas, sub-proyectos, proyectos y conjunto de proyectos en un Fondo de Recursos Compartido, ver Figura 6. 


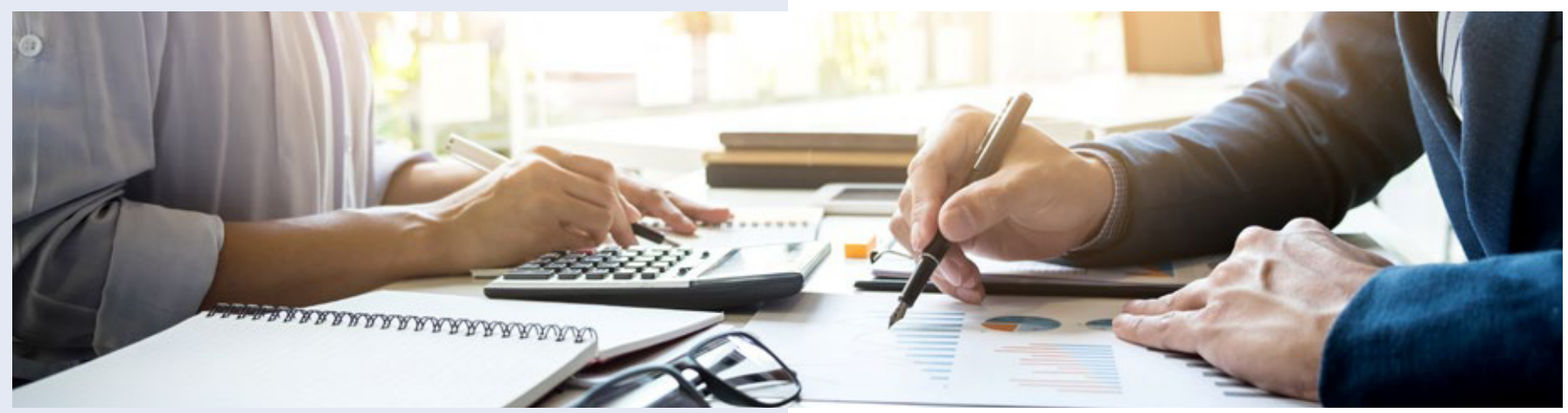

Por último, desde la perspectiva económica, el impacto de la propuesta se sustenta en el ahorro que supone tomar mejores decisiones con la información brindada por el modelo propuesto para el control de la ejecución de proyectos. Así como, ahorro de recursos, exportación de bienes y servicios útiles para el desarrollo de la sociedad de forma general.

\section{CONCLUSIONES}

El desarrollo de la evaluación estratégica de las empresas que trabajan por Proyectos en la DIP orientadas al BIM, tiene en su centro la gestión del conocimiento que genera el hombre, a través de la aplicación del modelo estratégico de la inversión, garantizando la mejora continua y el perfeccionamiento de la empresa inteligente de alto desempeño. El sistema de información integrado, estratégico, único y multidimensional para la gestión de los proyectos, en un modelo virtual en $3 \mathrm{D}$, brinda la información por cortes de los proyectos, con la evaluación de los indicadores en el tablero de comando del Cuadro de Mando Integral, para la toma de decisiones estratégicas, para la evaluación del desempeño de la organización.

El proceso de Inteligencia Empresarial se desarrolla de forma continua e integrada por niveles. Permite caracterizar la empresa a través de un estudio del comportamiento de los indicadores por etapas y desarrollar un diagnóstico para precisar el nivel de aplicación de los nuevos conceptos.

El ciclo de gestión de conocimiento crea las condiciones para compartir el conocimiento entre el equipo de trabajo y las partes interesadas, desarrolla hábitos y habilidades en la solución de los problemas, estimula la motivación por la elevación de la calidad del proceso y el sentido de pertenencia de sus integrantes, con el objetivo de obtener un mejor desempeño del recurso humano.

La metodología DIP cuenta con una buena experiencia de aplicación en múltiples empresas a través de asesorías, cursos de postgrado, diplomados, maestrías, especialidades y tutorías de doctorados. Dispone de contenidos en formato digital de libros de texto, multimedia y demos en Power Point narrados. Desarrolla una base de datos de datos de proyectos ejecutados con sus cronogramas de ejecución. Los cortes que permiten la fácil asimilación de los contenidos por los especialistas es un paso importante para la aplicación de la metodología BIM.

\section{BIBILIOGRAFÍA}

[1] Amendola L. (2004). Estratégicas y Tácticas en Dirección y Gestión de Proyectos. Project Management. Universidad Politécnica de Valencia.

[2] Bath, M. (2010). Project Classification Using Soft Computing. En: International Conference on Advances in Computing, Control \& Telecommunication Technologies, pp. 537-539.

[3] Certa, A., Enea, M., y Giallanza, A. (2010). A synthetic measure for the assessment of the project performance.

[4] Business Performance Measurement and Management, pp. 167-180. Springer-Verlag, Berlin.

[5] Delgado R. y Montes de Oca M. (2009). La gestión del Conocimiento Organizacional en la Toma de decisiones. En: Informática 2009. Palacio de Convenciones, La Habana, Cuba.

[6] Delgado, R. (2011). La Dirección Integrada de Proyecto como Centro del Sistema de Control de Gestión en el Ministerio del Poder Popular para la Comunicación y la Información. CENDA, Caracas, Venezuela.

[7] Espinosa A. (2012). La Ingeniería del valory la Constructibilidad. En: 16 Conferencia Científica. Palacio de Convenciones, La Habana, Cuba.

[8] Gao, H. (2010). A fuzzy-ANP approach to project management performance evaluation indices system. En:

[9] International Conference on Logistics Systems and Intelligent Management, IEEE, pp. 273-277.

[10] Govindarajan, A. (2014). A Novel Framework for Evaluating the Software Project Management Efficiency - An Artificial Intelligence Approach. TELKOMNIKA Indonesian Journal of Electrical Engineering, 12(9), 7054-7058.

[11] Holness, V. R. (2008). Building Information Modeling Gaining Momentum. ASHRAE Journal. pp. 28-40.

[12] Liu, Y., et al. (2013). Research on Evaluation of Project Management Maturity Model Based on BP Neural Network. Advances in Information Sciences and Service Sciences (AISS), 5(2), 693-701.

[13] Mewada, K. M., Sinhal, A., y Verma, B. (2013). Adaptive Neuro-Fuzzy Inference System (ANFIS) Based Software Evaluation. IJCSI International Journal of Computer Science, 10(1), 244-250.

[14] PMI (2017). A Guide to the Project Management Body of Knowledge. $\mathrm{PMBOK}^{\circledR}$ Guide, 6th Edition. Project Management Institute, Pennsylvania, EE.UU.

[15] Prince (2009). Managing Successful Projects with Prince2. In: TSO (Ed.), Londres.

[16] SEl (2010). CMMI para Desarrollo, Versión 1.3. Mejora de los procesos para el desarrollo de mejores productos y servicios. Technical Report, Software Engineering Institute, EE.UU.

[17] Stang, D. B. (2013). IT Project \& Portfolio Management Magic Quadrant. Gartner Inc., Stanford.

[18] Torres M. (2008). Fundamentos generales de la logística. Editora LogiCuba. 